\title{
Current flow mechanisms in $p-i-n$ structures based on cadmium telluride
}

\author{
P.M. Gorley *), M.V. Demych *), V.P. Makhniy ${ }^{*}$, Zs. J. Horvath ${ }^{* *}$, V.A. Shenderovsky ${ }^{* * *}$ \\ *) Chernivtsi National University, 2 Kotsyubynsky st., 58012 Chernivtsi, Ukraine, \\ phone: (+380 3725) 98473, (+3803722) 44221, e-mail: gorley@west.com.ua \\ ${ }_{* *}^{*}$ Research Institute for Technical Physics and Material Science, P.O.Box 49, 114 Budapest, H-1525 Hungary, \\ e-mail:horvzsj@mfa.kfki.hu \\ $\left.{ }^{* * *}\right)$ Physics Institute of National Academy of Science of Ukraine, 144 Prospekt Nauki, Kyiv 28, Ukraine, phone: (380-44) \\ 265 0777, fax: (380-44) 265 0777, e-mail: schender@iop.kiev.ua
}

\begin{abstract}
Electrical properties of p-i-n-structures obtained with low-temperature oxygen and lithium diffusion into low-resistive $\mathrm{n}$-CdTe substrates have been investigated. The role of generation-recombination processes as well as trapping, impact ionization and overbarrier carriers transport in the dark current formation of samples studies have been defined.
\end{abstract}

Keywords: p-i-n-structure, generation-recombination, injection, cut-off current, impact ionization, height of the barrier, cadmium telluride.

Paper received 16.06.00; revised manuscript received 26.11.01; accepted for publication 05.03.02.

\section{Introduction}

Cadmium telluride is still one of the most perspective materials for detectors of irradiation of optical and X-ray ranges [1]. The main part of these devices is mainly the diode structures of different type, which have many advantages comparing with resistive ones [2]. Simultaneously they have complicated characteristics of various types, which are being defined by the technology of barrier detector elaboration. Special place among these devices is given to the structures with high-resistive $i$-layer that improves some device parameters. In particular, in $m-i-n$-structures on the base of Au-CdTe contacts at $300 \mathrm{~K}$ it is possible to obtain photovoltaic efficiency of about 13\% [3]. These structures have specific electric properties, considered in [4]. Further we have discussed the dark current formation mechanisms of CdTe-diodes with $p-i-n$ structure.

\section{Device elaboration technology}

We have used CdTe monocrystals with conductivity of about $0.05 \Omega^{-1} \mathrm{~cm}^{-1}$ at $300 \mathrm{~K}$ as initial substrates. After mechanical and chemical polishing one of the biggest sides of the samples with dimensions of $4 \times 4 \times 1 \mathrm{~mm}^{3}$ was covered with In contacts. Opposite side was damped with water solution of lithium salt, after that the samples was kept in normal environmental conditions until the water from solution evaporates completely. Diffusion layer was created by annealing the samples in the air. Lithium and oxygen from the atmosphere, diffusing into $n$-CdTe lead to overcompensation of electron conductivity of the base crystal into hole one. These impurities could penetrate to different depth depending on the annealing temperature due to their different diffusion coefficients, solubility and different position of energy levels. Let us note that oxygen is rather deep acceptor $\left(E_{\mathrm{a}}=0.3 \mathrm{eV}\right)$ and leads to formation of $i$-area in 


\section{P.M. Gorley et al.: Current flow mechanisms in $p-i-n$ structures...}

n-CdTe [5]. Greater hole conductivity appears due to alloying with $\mathrm{Li}$, that forms shallow $\left(E_{\mathrm{a}}=0.014 \mathrm{eV}\right)$ levels. Changing annealing temperature and time it was possible to make diodes with $p-i$ - $n$-structure. Ohmic contact to the p-area was created by vacuum spraying of semitransparent golden film.

Capacitance of the structures studied depends very weakly over applied voltage $V$, which proves the presence of $i$-area. The thickness of the latter $d_{\mathrm{i}}$ depends on the diode fabrication conditions and under zero bias in the room temperature range has the value of $d_{\mathrm{i} 0}=30-50 \mu \mathrm{m}$. Rectifying coefficient of the structure at $300 \mathrm{~K}$ and $V=1 \mathrm{~V}$ was equal to $10^{5}$.



Fig. 1. CVC of surface-barrier diode (1) and $p$-i-n-structures (2) in the linearity region at $300 \mathrm{~K}$. On the inset - temperature dependence of the barrier height and base material resistance.

\section{Results and discussion}

The $p-i-n$-structures studied have much higher potential barrier than that of surface-barrier Au-CdTe diodes, though they are both grown over the same substrates. It is being illustrated by the data presented in the Fig. 1. Barrier height $\varphi_{o}$ could by easily found by extrapolation of linear regions of direct current-voltage curves (CVC) to their intersection with voltage axis, giving us $\varphi_{\mathrm{o}}=e V_{\mathrm{o}}$. The difference of barrier heights of $\Delta \varphi_{0} \approx 0,3 \mathrm{eV}$ for the mentioned structures is being caused by additional alloying of the surface layer of $i$-region with lithium. Due to this band bending at the boundary Au-CdTe for $p-i-n-$ diodes is significantly greater than that for $m-i$ - $n$-structures. The value $\varphi_{\mathrm{o}}$ of $p-i-n$-structures depends over the temperature $T$, decreasing with the increase of the latter (see inset to the Fig. 1). Let us note that temperature coefficient of barrier height changes $\gamma \varphi_{0} \approx 3,3 \cdot 10^{-2} \mathrm{eV} / \mathrm{K}$ is significantly greater than the temperature coefficient of the bandgap $E_{\mathrm{g}}$ for cadmium



Fig. 2. Initial regions of direct $\mathrm{CVC}$ branches of $p-i$ - $n$-structure at $300 \mathrm{~K}$ (1) and $370 \mathrm{~K}(2)$. On the inset - temperature dependence of recombination current and ideality coefficient.

telluride $\gamma_{E \mathrm{~g}} \approx 4,1 \cdot 10^{-4} \mathrm{eV} / \mathrm{K}$ [6]. It is caused mainly by shifting of Fermi level in $p$-region towards the middle ofthe bandgap with increase of $T$, as the specific resistivity of $n$-substrate practically does not depend on the temperature (inset to Fig. 1).

Initial regions of the straight branches of CVC (Fig. 2) are being well described with the expression that is characteristic to the generation-recombination processes in the space charge region (SCR) $[7,8]$ :

$$
I_{g r}=I_{g r}^{o}\left[\exp \left(\frac{e V}{n k T}\right)-1\right],
$$

where $I_{g r}^{o}$ - cut-off current at the voltage $V=0 \mathrm{v}$, and $n$ is the non-ideality coefficient of CVC. Experimental values of $n$ in the temperature ranges investigated within the experiment precision ranges are close to the value of 2.5 (inset to Fig. 2). This value is greater than expected $n=2$ for the case of recombination through single level, which is being predicted in Shockley-Hall-Read theory $[7,9]$. Dependence (1) with $n>2$ is being observed for the structures with high resistive region at the monotonous dependence of the injected carriers. If the thickness of $i$-region in this case is greater than diffusion length of electrons and holes $L$, then the ideality coefficient is being defined by the formula [8]: 


\section{P.M. Gorley et al.: Current flow mechanisms in p-i-n structures...}

$$
n \approx \operatorname{ch}\left(d_{i} / L\right)
$$

When $d_{\mathrm{i}}>L$ the value of $n$ would be greater than 2 , which is being observed. Taking the experimental value $n=2.5$, we will obtain the ratio $d_{\mathrm{i}} / L \approx 1.5$. For the given sample $d_{\text {io }} \approx 50 \mu \mathrm{m}$, which means that diffusion length takes the value of $L=33 \mu \mathrm{m}$ - it correlates well with the corresponding diffusion length $L_{\mathrm{n}}$ of electrons in cadmium telluride [6]. Let us note that $L_{\mathrm{p}}$ for CdTe crystals grown from melt, is significantly smaller and is about $0,1-2 \mu \mathrm{m}$ [10]. Increase of $L_{\mathrm{p}}$ could be reached in the material doped with chlorine [1], or in perfect epitaxial layers of $\mathrm{CdTe}$, which are grown at extra low temperatures.

Value of $I_{g r}^{o}$ depends on the material parameters and on the diode structure [7] :

$$
I_{g r}^{o}=\frac{e d_{i o}}{\sqrt{\tau_{n} \tau_{p}}} \sqrt{N_{c} N_{v}} \exp \left(-\frac{E_{a}}{n k T}\right)
$$

where $t_{n}$ and $t_{p}$ - lifetimes of electrons and holes respectively to the deep centers, $\mathrm{N}_{\mathrm{c}}$ and $\mathrm{N}_{\mathrm{v}}$ - carrier effective densities of states in the conduction and valence bands, and other designations are common. Temperature dependence of $I_{g r}^{o}$ is being defined mainly by the exponential term and has a good correlation with experimental data (inset to Fig. 2). The activation energy $E_{a}$ could be easily found from the inclination of the line and is $1.6 \mathrm{eV}$, which corresponds to bandgap of CdTe at $0 \mathrm{~K}$ [6].

At the forward bias $V \geq 0.6 \mathrm{~V}$ dependence $I(V)$ becomes more weaker in comparison with the exponential one, which is given by (1) (see also fig. 2). It is caused by ideality lost of one or both injecting junctions that could be caused by the process of non-equilibrium carriers trapping by the deep trap levels. In this case CVC is being described by power low

$$
I \sim V^{m} \text {. }
$$

The power m could take values $3 / 2,2$ or 4 depending on the role of the diode structure parts involved into recombination processes. In our case experimental CVC are in good correlation with (4), if we put $m=4$. The same dependencies for the first time was observed in [11] without any explanations while investigating $p$ - $i$ - $n$-structures based on Ge. These CVC then were explained theoretically in [12] taking into account sticking centers. Energy position of the latter could be defined from the temperature dependence of the current on the power region of the curve at $V=$ const [13]. As it is seen from the data of Fig. 4 (inset), the experimental dependence $I(T)$ is being well-approximated with the straight line in the reference system $\ln I-10^{3} / T$. Activation energy has the value of about $0.35 \mathrm{eV}$ and could refer to the position of impurity level of oxygen, which are forming highresistive $i$-region. Diode structures of this kind show also linear decrease of the voltage $V_{\mathrm{o}}$, which is being defined as cut-off of the straight lines $I^{1 / 4}-V$ at $I^{1 / 4}=0$ (Fig. 3). Experimental dependencies $V_{\mathrm{o}}(T)$ of the studied $p-i$ - $n$-structures fits well with the straight line (inset to Fig. 3). These data allows to calculate the bandgap of the material with the expression [8]:

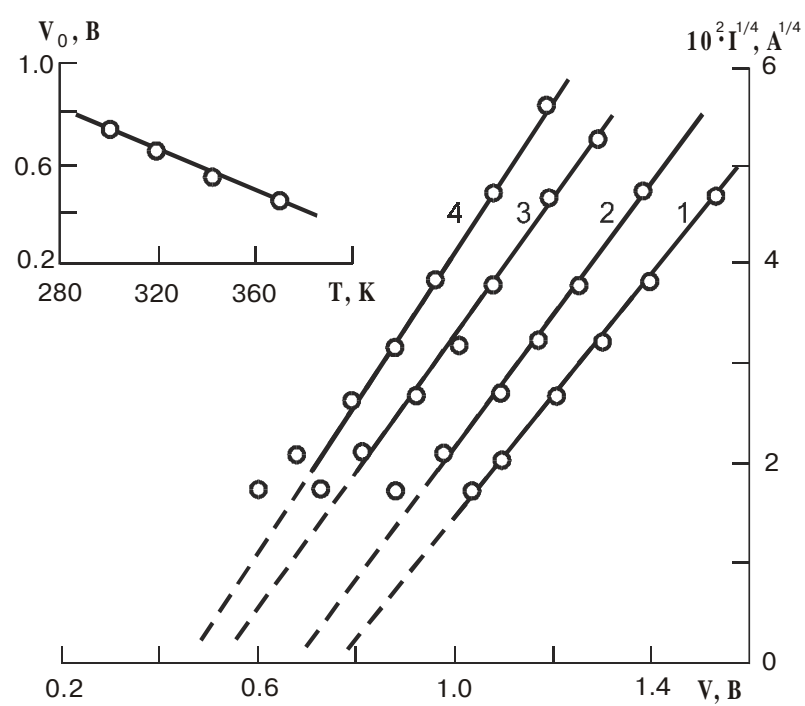

Fig. 3. Direct branches of $p-i-n$-structure $C V C$ on the power regions at temperatures of 300 (1), 320 (2), 340 (3) and $370 \mathrm{~K}$ (4). On the inset - temperature dependence of cut-off voltage $V_{\mathrm{o}}$.



Fig. 4. Comparison of direct CVC branches of $p-i$ - $n$-structures with expression (7) under 300 (1), 320 (2), 340 (3) and $370 \mathrm{~K}$ (4). On the inset - temperature dependences of the overbarier cutoff current $I_{\mathrm{s}}$ and direct current at $V=1 \mathrm{~V}$.

$$
\left.E_{g}=e \mid V_{o}\left(T_{1}\right) T_{1}^{-1}-V_{o}\left(T_{2}\right) T_{2}^{-1}\right] /\left(T_{1}^{-1}-T_{2}^{-1}\right)(5)
$$

The bandgap value $E_{\mathrm{g}}$ obtained with (5) appears equal to $1.7 \mathrm{eV}$, that correlates with bandgap of CdTe taking into account the precision of the experimental measurements.

When forward bias reaches the values that are close to the compensation of the injecting junctions, then at last over-barrier current $I_{\mathrm{d}}$ becomes dominating. In general it is given by the formula [14]: 


$$
I_{d}=I_{s} \exp \left(e V_{b} / k T\right)
$$

where $I_{\mathrm{s}}$ - cut-off current at $V_{\mathrm{b}}=0 \mathrm{~V}$. Voltage of the barrier area $V_{\mathrm{b}}$ is not equal to the applied voltage $V$, because it partly dissipates over the series resistance $R_{\mathrm{O}}$ of the diode material and his ohmic contacts. Therefore, $V_{b}=V-I_{d} R_{o}$, and (6) could be presented in the form [10]:

$$
\ln I_{d}-\frac{e V}{k T}=\ln I_{s}-\frac{e R_{o}}{k T} I_{d}
$$

At practice we measure full current $I$, that is not equal in general case to over-barrier one. But, in the region of high biases close to $\varphi_{\mathrm{o}} / e$, with the sufficient precision it is possible to consider $I_{\mathrm{d}}=I$ and to perform the respective changes in (7). The latter is well-observed at the experiment, graphically representing the straight line in the ranges of $I, V$ and $T$, where over-barrier carrier transport process are dominating (Fig. 4). Approximation of the linear ranges of the dependence (7) to their intersection with the $I$-axis for $I=0$ corresponds to the logarithm of the saturation current. As $I_{s} \sim \exp \left(-\varphi_{o} / k T\right)[14]$, then its temperature dependence is being determined by the barrier height $\varphi_{\mathrm{o}}$, which is the sum of the barrier heights of $p-i$ and $i$ - $n$ - junctions. Mentioned dependence has good experimental proof, but the value $\varphi_{\mathrm{o}}(0) \approx 2.5 \mathrm{eV}$ is significantly greater the maximal possible one for $\mathrm{CdTe}$ bandgap of $E_{\mathrm{g}}(0) \approx 1.6 \mathrm{eV}$. This contradiction could be removed taking into account anomalously great value of $\gamma \varphi_{\mathrm{o}}$ for the structures studied. Admitting linear dependence of the barrier height over the temperature [14]:

$$
\varphi_{o}(T)=\varphi_{o}(0)-\gamma_{\varphi_{o}} T
$$

One could obtain good coincidence of calculated and experimental value of $\varphi_{\mathrm{o}}$ in the considered ranges of $T$.

It is logical to assume that generation-recombination processes could show up either in the case of reverse bias, especially under low voltage. It is being illustrated by the data, presented in Fig. 5. At $|e V| \geq 3 n k T$ the reverse current $I_{g r}^{r e v}=\frac{d_{i}}{d_{i o}} I_{g r}^{o}$, and its dependence over the voltage is being defined with term $d_{\mathrm{i}}$. The analysis shows that better coincidence with the experimental data is being achieved if we assume that $d_{i}=d_{i o} \sqrt{\varphi_{o}-e V}$, when the barrier is considered to be abrupt. (Let us stress that parameter $d_{\mathrm{io}}$ in (3) for $I_{g r}^{o}$ corresponds to $d_{\mathrm{i}}$ at $V=0$ ). This assumption made is quite natural, because the carrier concentration in $p$ - and $n$-regions are greater than that in $i$-region. As a result the SCR is located in this region, while $p$ - $i$ - and $i$ - $n$ - junctions are abrupt. To calculate reverse generation current we used the formula

$$
I_{g r}^{r e v}=I_{g r}^{o} \sqrt{\varphi_{o}-e V}
$$

The parameters necessary to find $I_{g r}^{o}$ and $\varphi_{\mathrm{o}}$ were taken from the straight branches of CVC for the corresponding $T$. We have proved our calculations to be correct also with temperature dependence of reverse current under the range of voltage, when generation-recombination processes are dominating. It is being illustrated by data, presented on the inset to Fig. 5, which fit well with exponential dependence with the power $E_{a} / n k T$. Activation energy $E_{a}$ correlates with $E_{\mathrm{g}}$ of CdTe at $0 \mathrm{~K}$, when

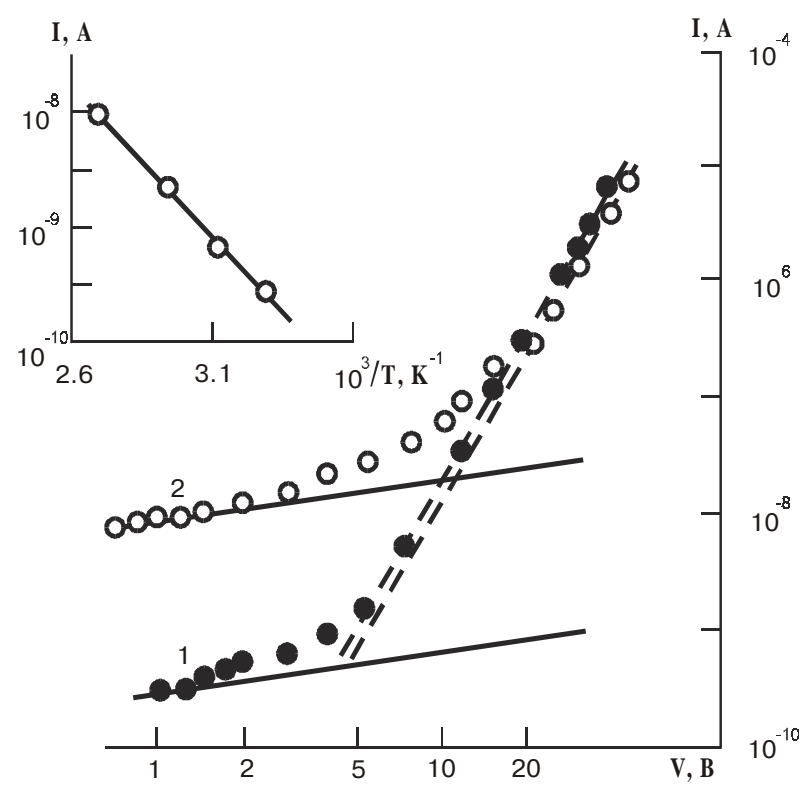

Fig. 5. Reverse branches of CVC for $p-i-n$-structure at the temperatures of $300 \mathrm{~K}$ (1) i $370 \mathrm{~K}$ (2). Dots represents the experimental data, solid lines - our calculations due to formula (9). On the inset - temperature dependence of reverse current at $V=1 \mathrm{~V}$.

the ideality coefficient is being considered to have the value of 2.5, as it was for direct bias. Intensity of generation-recombination processes due to the theory [7-9] should grow with temperature increase, and the contribution of $I_{g r}^{o}$ into the full current should, respectively, increase. It is completely corresponds to the experimental data, that proves the fact that initial regions of straight-line direct and reversed bias CVC branches of the objects studied are being defined with generation-recombination processes in $i$-region of the structures.

Deviation of experimental points from the calculated dependence $I(V)$ at $V_{r e v} \geq 2-5 \mathrm{~V}$ is connected with other current transfer mechanism. The analysis shows that it is caused by avalanche multiplication of carriers due to impact ionization. Negative temperature coefficient of breakdown voltage could be considered as direct proof of the named process [14]. As it is seen from Fig. 5, at $V_{\text {rev }} \geq 20 \mathrm{~V}$ the current at higher $T$ is smaller than that at lower ones. It is caused by the fact that temperature growth leads to increase of oscillation intensity of crystal lattice atoms as well as the possibility of carrier scattering over them. As a result the threshold energy of impact ionization could be acquired by the carrier while crossing bigger distance, than the free-run distance that needs higher voltage. Let us also note that additional evidence of the avalanche processes is photocurrent multiplication for the bigger reverse voltages as a result of illumination of $p-i$ - $n$-structures with quanta from fundamental absorption range. 
P.M. Gorley et al.: Current flow mechanisms in p-i-n structures...

\section{Conclusion}

In the given paper it was found that initial regions of direct and reverse $\mathrm{CVC}$ are being defined by generationrecombination processes in $i$-region of the structure under monotonous dependence of the carriers injected in it. The power dependence of direct current over the voltage at $V \geq 0.6 \mathrm{~V}$ is being caused by trapping processes of the deep traps with energy of $\sim 0,35 \mathrm{eV}$. Overbarrier current appears only under direct voltages with values close to the height of potential barrier of the structure. Quick growth of reverse current at $V \geq 2-5 \mathrm{~V}$ is caused by the avalanche multiplication of the carriers due to the process of impact ionization. Therefore, for the studied $p-i-n-$ structures it is characteristic to have various mechanisms of current transfer, which is caused by tight interconnection of contact and bulk phenomena.

\section{References}

1. D.V. Korbutyak, S.V. Mel'nychuk, E.V. Korbut, M.M Borysyuk // Cadmium telluride: impurity-defect states and detector properties. - Kyiv: Ivan Fedorov, 198 p., (2000).

2. V.P. Makhniy, V.E. Baranyuk, Ya.M. Barasyuk, M.V. Demych, O.V. Makhniy, V.V. Mel'nyk, B.M. Sobishchans'ky, Barrier detectors of electromagnetic irradiation on the base of wide-band II-VI compounds // Scientific messenger of Chernivtsi National University, 66, Physics. Electronics.- Chernivtsi: Chernivtsi National University, pp.24-27, (1999).
3. P.M. Gorley, M.V. Demych, V.P. Makhniy, Z. Swiątek, K.S. Ulyanitsky, R. Ciach, Photoelectric properties of metal - cadmium telluride contacts with modified surface // Scientific messenger of Chernivtsi National University, 63 Physics. Electronics.- Chernivtsi: Chernivtsi National University, pp.82-84, (1999).

4. V.P. Makhniy, M.V. Demych, P.M. Gorley, P.P. Horley, Zs.J.Horvath, Vo Van Tuyen, Electrical properties of photoconverters on the base of Au-CdTe structures // Abstr. Joint Meeting European Conf. on Photovoltaics. 25-27 October 1999. Cracow-Poland. 19 p. (1999).

5. Cadmium telluride // Edited by B.M. Wool - Moscow: Nauka, p. 142 (1968)

6. N.N. Berchenko, V.E. Krevs, V. G. Sredin // Semiconductor solid solutions and their applications - Moscow: Voenizdat (1982).

7. C.T. Sah, R.N. Noyce, W. Shockley, Carrier Generation and Recombination in p-n-Junction and p-n-Junction Characteristics // Proc. IRE.- 45.- pp. 1228-1243 (1975).

8. E.I. Adirovich, P.M. Karageorgiy Alkalaev, A.Yu. Leiderman, // Currents of double injection in semiconductorsMoscow: Soviet Radio, 320 p. (1978).

9. W. Shockley, W.T. Read, Statistic of the Recombination of Holes and Electrons // Phys. Rev.- 87, - pp. 835-849 (1952).

10. V.P. Makhniy, Physical processes in diode structures on the base of wide-band compounds $A^{2} B^{6} / /$ Thesis for Doctor's degree in Physics and Mathematics - Chernivtsi, 293 p. (1992).

11 , N.H. Fletcher The high current limit for semiconductor junction devices // Proc. IRE.- 45, N6.- pp. 862-872 (1957).

12. A.Yu. Leyderman // Influence of sticking centers on the current characteristic of semiconductor $p$ - $n$ - $n+$-diodell FTP.- 3, 10 pp. 1492-1505 (1969).

13. M. Lampert, P. Mark // Injection currents in the solid state / Russian translation over supervision of S.M. Ryvkin.- Moscow: Mir, 416 p. (1973).

14. S.M. Sze // Physics of Semiconductor Devices, $2^{\text {nd }}$ edition $-J$. Wiley \& Sons, New York, 868 p. (1981). 